\title{
Cough Impairment and Risk of Postoperative Pulmonary Complications After Open Upper Abdominal Surgery
}

\author{
Daniela B Bonfim Colucci MSc, Julio F Fiore Jr PhD, Denise M Paisani PhD, \\ Thais Telles Risso PhD, Marcelo Colucci MSc, Luciana Dias Chiavegato PhD, \\ and Sonia Maria Faresin PhD
}

\begin{abstract}
BACKGROUND: Cough impairment is often described as part of the pathophysiological basis of postoperative pulmonary complications (PPCs). However, there have been few studies examining cough effectiveness and its relationship with PPCs following open upper abdominal surgery. The goal of this study was to estimate (1) changes in cough efficacy after upper abdominal surgery through the assessment of peak cough flow and (2) the extent to which cough impairment is associated with postoperative pain, FVC, and risk of PPCs. METHODS: This prospective cohort study assessed 101 subjects $(45 \%$ male, $56 \pm 16$ y old) admitted for elective upper abdominal surgery. Measurements of peak cough flow and FVC were performed on the day before surgery and repeated on postoperative days 1,3 , and 5. PPCs were assessed daily by a pulmonologist blinded to the cough measurement results. RESULTS: Peak cough flow dropped to 54\% of the preoperative value on postoperative day 1 and gradually increased on postoperative days $3(65 \%)$ and $5(72 \%)$ $(P<.05)$. On all postoperative days, peak cough flow was strongly correlated with FVC $(P<.001)$ and weakly correlated with pain $(P=.006)$. Six subjects $(6 \%)$ developed PPCs. The association between peak cough flow and risk of PPCs was not statistically significant (unadjusted odds ratio of $0.80,95 \%$ CI $0.45-1.40, P=.44$; adjusted odds ratio of $0.66,95 \%$ CI $0.32-1.38, P=.41$ ). CONCLUSIONS: Cough effectiveness is impaired after upper abdominal surgery. Postoperative restrictive lung dysfunction seems to be associated with this impairment. There is no significant association between peak cough flow and PPCs; however, cough impairment might result in clinically important consequences in a high-risk population. Key words: cough; risk factors; surgery; postoperative complications; postoperative care. [Respir Care 2015;60(5):673-678. (c) 2015 Daedalus Enterprises]
\end{abstract}

\section{Introduction}

Postoperative pulmonary complications (PPCs) are an important cause of morbidity following upper abdominal

Ms Colucci and Dr Faresin are affiliated with the Respiratory Department, Federal University of São Paulo, São Paulo, Brazil. Dr Fiore is affiliated with the Steinberg-Bernstein Centre for Minimally Invasive Surgery and Innovation, McGill University Health Centre, Montreal, Quebec, Canada. Dr Paisani is affiliated with the Physical Therapy Department, School of Medicine, University of São Paulo, São Paulo, Brazil. Dr Risso is affiliated with the Physical Therapy Department, University Center of Vila Velha, Vila Velha, Espírito Santo, Brazil. Mr Colucci is affiliated with the Physical Therapy Department, Nove de Julho University, São Paulo, Brazil. Dr Chiavegato is affiliated with the Master's and Doctoral Program, University City of São Paulo (UNICID), São Paulo, Brazil. surgery and contribute to significant increases in hospital length of stay and health-care costs. ${ }^{1}$ A patient's inability to cough effectively after surgery is often described as part of the pathophysiological basis of PPCs, as it may lead to excessive accumulation of pulmonary secretions and increase the risk of obstructive atelectasis and respiratory

\footnotetext{
This work was supported by the Coordenação de Aperfeiçoamento de Pessoal de Nível Superior (CAPES), Brazil. The authors have disclosed no conflicts of interest.

Correspondence: Daniela B Bonfim Colucci MSc, Rua Borges Lagoa, 760-3 andar, 04023-900 São Paulo, Brazil. E-mail: daniela.bbonfim@ terra.com.br.
}

DOI: $10.4187 /$ respcare. 03600 
infections. ${ }^{2}$ Postoperative pain and restrictive lung dysfunction are believed to be important factors associated with cough impairment. ${ }^{2}$

Despite the relevance of this topic for clinicians involved in the respiratory care of patients undergoing upper abdominal surgery, there have been few studies examining cough efficacy in this surgical population. Also, there is still no empirical evidence that cough impairment is a risk factor for PPCs. In this study, we aimed to quantify changes in cough efficacy after upper abdominal surgery through assessment of peak cough flow and estimate the extent to which cough impairment is associated with postoperative pain, FVC, and PPC risk.

\section{Methods}

\section{Design}

This was a prospective cohort study. Our protocol was approved by the local ethics committee, and all subjects provided written informed consent.

\section{Subjects}

Adult patients ( $\geq 18 \mathrm{y}$ old) admitted for elective upper abdominal surgery at a university tertiary hospital were considered for inclusion. Upper abdominal surgery was defined as any surgical procedure performed through an incision above or extending above the umbilicus. ${ }^{3}$ The exclusion criteria included neuromuscular or cognitive diseases, inability to perform the proposed measures, or previous abdominal surgery. Subjects were withdrawn from the study in cases of surgery cancellation, orotracheal intubation for $>24 \mathrm{~h}$, surgery through a thoracic approach, intraoperative death, or reoperation.

\section{Procedures}

All subjects underwent standardized preoperative assessment, including complete medical history and physical examination. Clinical diagnosis of chronic respiratory disease (eg, COPD, asthma) or presence of respiratory symptoms (eg, dyspnea, cough, mucus retention) was considered a respiratory comorbidity. Measurement of peak cough flow was performed on the day before surgery and repeated on postoperative days 1, 3, and 5. Pain assessment and complete spirometry were performed at the same time intervals after the cough evaluation. Development of PPCs was assessed daily by a pulmonary physician who was blinded to the cough measurement results. Subjects were treated in a traditional postoperative care environment (ie, we did not use an enhanced recovery protocol at the time of data collection) and underwent standardized physiotherapy sessions (breathing exercises and ambulation) daily

\section{QUICK LOOK}

\section{Current knowledge}

Postoperative pulmonary complications (PPCs) are common and costly. PPCs are a leading cause of morbidity and mortality following upper abdominal surgery and contribute to prolonged hospital stays and costs. Ineffective cough secondary to pain can lead to retained secretions and reduced lung volumes, promoting atelectasis.

\section{What this paper contributes to our knowledge}

Cough efficacy was significantly impaired following upper abdominal surgery. Postoperative restrictive lung dysfunction was associated with impaired cough, but there was no association between the measured peak cough flow and development of a PPC.

from postoperative day 1 until hospital discharge. During the period of data collection, opioid-based patient-controlled analgesia was the standard pain management regimen used in our institution.

\section{Cough Measurement}

Peak cough flow is considered a relevant measure of cough efficacy, as mucus clearance is largely dependent on the magnitude of expiratory flow during cough. ${ }^{4}$ This measure can be accurately taken at the bedside using portable devices such as handheld spirometers or flow meters. ${ }^{5,6}$ In this study, peak cough flow was measured with a portable spirometer (MIR Spirobank, Rome, Italy) connected to an oronasal mask (Adult 5, Vital Signs, Totowa, New Jersey). All measures were taken by a trained physiotherapist using the technique described by Fiore et al. ${ }^{6}$ Subjects sat in a semirecumbent position $\left(60^{\circ}\right)$ and were instructed to "take a deep breath and cough as forcefully as possible." Five measures were performed with intervals of $30 \mathrm{~s}$ between each. Therefore, the 3 highest peak cough flow values were considered for analysis. The spirometer was calibrated before each evaluation day according to the manufacturer's recommendation. The assessor was responsible for adjusting the mask on the subject's face to prevent air leakage. Pain during cough was evaluated using a visual numeric scale graduated from 0 (minimum) to 10 (maximum $)^{7}$ after each measurement session. After cough measurement, spirometry was performed according to American Thoracic Society recommendations. ${ }^{8}$ FVC was the main lung function variable of interest in this study, as reduced FVC is considered a proxy for restrictive lung dysfunction. ${ }^{9}$ 


\section{Cough Impairment After Open Upper Abdominal Surgery}

\section{Postoperative Pulmonary Complications}

Subjects were followed for development of PPCs from the day of surgery to hospital discharge. Postoperative pulmonary complications were defined by the occurrence of one or more of the following ${ }^{10,11}$ : (1) pneumonia, the presence of radiological evidence of pulmonary infiltration associated with at least 2 of the following criteria: purulent sputum, elevated body temperature $\left(>38.0^{\circ} \mathrm{C}\right)$, and leukocytosis ( $\geq 25 \%$ above baseline preoperative value); (2) tracheobronchitis, a marked increase in sputum production or presence of purulent sputum in a subject with a normal chest $\mathrm{x}$-ray; (3) atelectasis with clinical repercussion, radiological evidence of atelectasis associated with dyspnea; (4) acute respiratory failure, acute deficiency of gas exchange with necessity for invasive or noninvasive mechanical ventilation; and (5) bronchoconstriction, the presence of wheezing associated with dyspnea requiring bronchodilator prescription or change in preoperative bronchodilator dosage.

\section{Statistical Analysis}

The sample size requirement for this study was calculated based on the results of our pilot data $(n=18)$ showing a difference of $1.5 \mathrm{~L} / \mathrm{s}(\mathrm{SD}$ of $1.7 \mathrm{~L} / \mathrm{s})$ in peak cough flow between subjects who developed PPCs and those who did not. We used a formula for unequal group sizes ${ }^{12}$ expecting an incidence of PPCs of $14 \% .^{10}$ According to this calculation, a sample of 100 subjects (13 with PPCs and 87 without PPCs) was necessary to provide a statistical power of $80 \%$ with a 2 -sided .05 significance level. Data analysis was performed using Stata 12.0 (StataCorp, College Station, Texas). Peak cough flow data were treated as normally distributed as per analysis of skewness and kurtosis. One-way analysis of variance (repeated measures) was used to compare peak cough flow across the 4 measurement times (preoperative and postoperative days 1, 3, and 5). We used paired $t$ tests with adjustment for multiple comparisons (Bonferroni approach) to assess pairwise differences. The relationship between peak cough flow with pain and FVC on each postoperative day was examined using Pearson correlation coefficients (r). The strength of correlations was classified as very weak $(r=0-0.19)$, weak $(r=0.2-0.39)$, moderate $(r=0.40-0.59)$, strong $(\mathrm{r}=0.6-0.79)$, and very strong $(\mathrm{r}=0.8-1) .{ }^{13}$ We used logistic regression to estimate the association between peak cough flow on postoperative day 1 and risk of PPCs. Adjustments were made for potential confounders (preoperative peak cough flow, sex, age, body mass index, and presence of respiratory comorbidities). Odds ratios were interpreted as the impact of a 1-unit increase in peak cough flow on the odds of developing PPCs. Cough measures obtained on postoperative days 3 and 5 were not included
Table 1. Characteristics of Subjects

\begin{tabular}{lc}
\hline \multicolumn{1}{c}{ Characteristic } & Values \\
\hline Subjects, N & 101 \\
Age, mean $\pm \mathrm{SD}, \mathrm{y}$ & $56 \pm 16$ \\
Males, $n(\%)$ & $45(45)$ \\
Respiratory comorbidity, $n(\%)$ & $15(15)$ \\
Current smoker, $n(\%)$ & $32(32)$ \\
Preoperative FVC, mean $\pm \mathrm{SD}, \%$ predicted & $94 \pm 26$ \\
Preoperative FEV, mean $\pm \mathrm{SD}, \%$ predicted & $92 \pm 25$ \\
Preoperative peak cough flow, mean $\pm \mathrm{SD}, \mathrm{L} / \mathrm{s}$ & $6.40 \pm 2.09$ \\
Type of surgery, $n(\%)$ & $32(32)$ \\
Colorectal & $21(21)$ \\
Gastric & $11(11)$ \\
Hepatobiliary & $7(7)$ \\
Esophageal & $4(4)$ \\
Pancreatic & $20(20)$ \\
Exploratory laparotomy & $6(6)$ \\
Hernia repair & $372 \pm 123$ \\
Time of anesthesia, mean $\pm \mathrm{SD}$, min & \\
Type of postoperative analgesia, $n(\%)$ & $71(70)$ \\
Intravenous & $30(30)$ \\
Epidural & \\
\hline
\end{tabular}

in the regression analysis due to potential for bias (lower peak cough flow in these measurement times can be a consequence of the presence of PPCs and not a risk factor for their development). Results were considered statistically significant when $P \leq .05$ and/or 95\% CIs did not include 1. For pairwise comparisons of peak cough flow across the 4 measurement times, $P<.008$ was considered statistically significant after the Bonferroni correction.

\section{Results}

Of 130 consecutive subjects screened over a period of $2 \mathrm{y}, 101$ were included in the analysis. Three subjects were excluded because of cognitive deficits. Twenty-five subjects were withdrawn from the study after initial assessment because of surgery cancellation $(n=7)$, laparoscopic approach $(n=4)$, or mechanical ventilation for $>24 \mathrm{~h}$ $(n=14)$. One patient refused to perform the postoperative assessment. The characteristics of the subjects are summarized in Table 1.

Figure 1 shows the progression of peak cough flow over the perioperative period. Peak cough flow dropped to 54\% of the preoperative value on postoperative day 1 $(6.40 \pm 2.09 \mathrm{~L} / \mathrm{s}$ vs $3.41 \pm 1.53 \mathrm{~L} / \mathrm{s}, P<.001)$ and gradually increased on postoperative day $3(4.18 \pm 0.92 \mathrm{~L} / \mathrm{s}$, $P<.001)$ and postoperative day $5(4.69 \pm 1.92 \mathrm{~L} / \mathrm{s}$, $P<.001$ ). In the last measurement time (postoperative day 5), peak cough flow was still significantly lower (72\%) in relation to the preoperative value $(P<.001)$. We found a strong positive correlation between peak cough flow and 


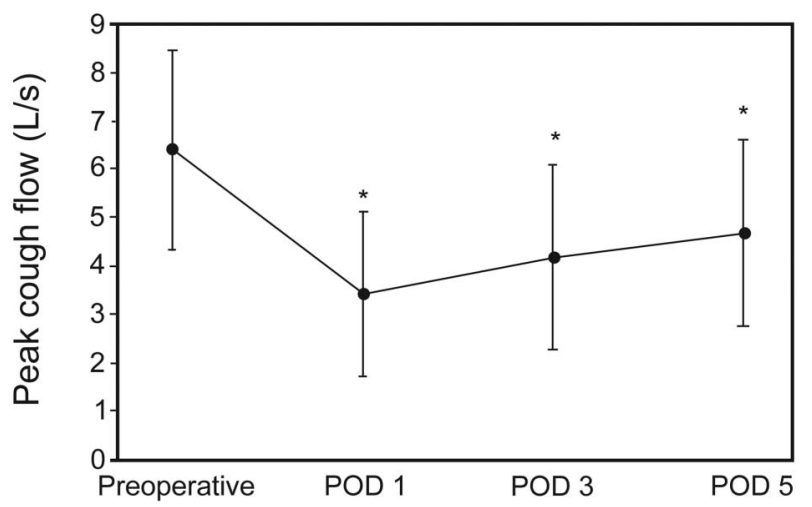

Fig. 1. Progression of peak cough flow over the perioperative period. $\mathrm{POD}=$ postoperative day. ${ }^{*} P<.001$ compared with previous measurements. Data are shown as mean \pm SD.

Table 2. Univariate and Multivariate Logistic Regression Estimating the Relationship Between Peak Cough Flow on Postoperative Day 1 and Risk of Postoperative Pulmonary Complications

\begin{tabular}{lcc}
\hline \hline & Odds ratio $(95 \% \mathrm{CI})$ & $P$ \\
\hline Univariate analysis & $0.80(0.45-1.40)$ & .44 \\
Multivariate analysis $\dagger$ & $0.66(0.32-1.38)$ & .41
\end{tabular}

$\lceil$ Multivariate analysis $w a s$ adjusted for preoperative peak cough flow, sex, age, body mass index, and presence of respiratory comorbidities.

FVC on all postoperative days (postoperative day 1: $\mathrm{r}=0.74, P<.001$; postoperative day $3: \mathrm{r}=0.80, P<.001$; postoperative day $5: \mathrm{r}=0.77, P<.001)$. Peak cough flow was negatively correlated with pain scores, but the correlation was weak (postoperative day $1: \mathrm{r}=-0.27, P=.005$; postoperative day $2: \mathrm{r}=-0.27, P=.002$; postoperative day 5: $\mathrm{r}=-0.34, P=.006$ ).

Postoperative pulmonary complications were diagnosed in 6 subjects $(6 \%)$. Logistic regression results are presented in Table 2. The association between peak cough flow on postoperative day 1 and risk of PPCs was not statistically significant in the unadjusted analysis (odds ratio of $0.80,95 \%$ CI $0.45-1.40, P=.44$ ) or in the adjusted analysis (odds ratio of $0.66,95 \%$ CI $0.32-1.38$, $P=.41)$.

\section{Discussion}

The results from this prospective cohort study confirm the hypothesis that cough efficacy is impaired in the postoperative period following upper abdominal surgery. Peak cough flow dropped to $54 \%$ of the preoperative value on postoperative day 1 . On postoperative day 5 , peak cough flow was still significantly lower $(72 \%)$ in relation to the measurements observed before surgery. Peak cough flow was strongly correlated with FVC and weakly correlated with pain during cough. Six subjects in our cohort (6\%) developed PPCs. Lower peak cough flow on postoperative day 1 tended to increase the risk of PPCs without reaching statistical significance.

A major strength of this study was that it involved a large sample of subjects undergoing open upper abdominal surgery; therefore, our results are likely to be representative of peak cough flow progression in the postoperative period. Consecutive subjects undergoing a wide variety of procedures were included to avoid sampling bias. We used data that were prospectively collected in a standardized manner, which potentially reduced variance in the quality of information recorded. The criteria used to define PPCs were rigorous and clinically relevant in relation to impact on morbidity and hospital stay. ${ }^{10,11}$

This study has the limitations of observational studies in attributing causality and controlling for confounders. As only subjects undergoing open upper abdominal surgery were included in the analysis, our results cannot be extrapolated to lower abdominal procedures or laparoscopic surgery. Another important limitation that needs to be noted is that the incidence of PPCs in our cohort may not have been large enough to ensure that the logistic regression analysis had adequate statistical power. We performed an a priori sample size estimation based on the incidence of PPCs observed in a previous study by our research group $(14 \%)^{10}$; however, the incidence found in the current study was lower (6\%). As both studies were conducted in the same surgical unit, involved similar populations, and used the same criteria to define PPCs, we hypothesize that the lower incidence is attributed to changes in perioperative care in recent years (ie, use of more aggressive regimens of early mobilization and respiratory physiotherapy, improvements in postoperative analgesia, and selective use of nasogastric tubes). We cannot exclude the possibility that the association between peak cough flow and risk of PPCs could become significant if the sample size was increased.

The incidence of PPCs after upper abdominal surgery reported in the literature has been widely variable. In a review spanning 1980-2005, Smetana et $\mathrm{al}^{1}$ identified 9 studies focused on open abdominal surgeries with incidence of PPCs ranging from $0.9 \%$ to $69.5 \%$. This variability was attributed to differences in criteria used to define PPCs, assessment times, and inclusion/exclusion criteria. The incidence of PPCs observed in our study is comparable to that (7\%) observed by Smith et $\mathrm{al}^{14}$ and Canet et al, ${ }^{15}$ who used similar diagnostic criteria. A study by our research group also found similar PPC rates (7.2\%). ${ }^{16}$

To the best of our knowledge, our study is the first to measure the influence of upper abdominal surgery on cough efficacy and to assess the relationship of peak cough flow with incision pain, FVC, and risk of PPCs. Previous liter- 
ature assessing cough function in this patient population was limited to a small study $(n=24)$ that demonstrated that the threshold for triggering a reflex cough by chemical irritation (capsicum and citric acid) was significantly increased on postoperative day 1 and returned to preoperative values on postoperative day $4 .{ }^{17}$ The authors speculated that the use of opioid analgesia may be mainly responsible for suppression of the cough reflex. There is also limited literature evaluating postoperative cough in other surgical populations. In a study of subjects undergoing thoracotomy $(n=24)$, cough reflex and intrapleural pressure during cough were reduced in the immediate postoperative period. ${ }^{18}$ Subjects who developed PPCs (chest infection) tended to have lower cough intrapleural pressure, but, as in our study, the difference did not reach statistical significance. As these studies were published $>20$ y ago, caution should be taken to generalize their findings to the current time.

It has been suggested that restrictive lung dysfunction and postoperative pain are important factors leading to reduced cough efficacy after surgery. ${ }^{2}$ In the present study, peak cough flow was strongly correlated with FVC and weakly correlated with incision pain. This finding suggests that the presence of restrictive lung dysfunction may be an important factor leading to impaired cough efficacy after surgery. Reduction of lung volume due to general anesthesia, diaphragmatic dysfunction, and abdominal distention is believed to be the cause of this restrictive pattern. ${ }^{19,20} \mathrm{We}$ speculate that reduction of lung volume impairs cough efficacy by altering the length-tension relationship of expiratory muscles and decreasing pleural and lung recoil pressures during cough. Although Tokuda et $\mathrm{al}^{21}$ showed improvement in peak cough flow during the use of transcutaneous electrical nerve stimulation for treatment of postoperative pain in this population, in our study, the correlation between incision pain and peak cough flow was significant but weak. This result may have been influenced by the subjectivity of pain measurements, small sample size, and preoperative individual characteristics.

Cough techniques are often included in the management of postoperative patients to improve cough efficacy and reduce the risk of PPCs. After abdominal surgery, it is a common recommendation that patients cough following a maximum inspiration while supporting their incision, repeating the technique at regular intervals (eg, every hour). ${ }^{22}$ Overall, our results do not support that reduced cough efficacy is a significant contributor for risk of PPCs. This questions the need for implementing strategies to improve cough in the postoperative period. Several randomized controlled trials have evaluated cough techniques as part of a comprehensive physiotherapy treatment of patients undergoing abdominal surgery ${ }^{23}$; however, these studies were often methodologically flawed, and results were inconsistent. Mackay et $\mathrm{al}^{24}$ found no significant differences in
PPC rates of subjects who received cough instructions as part of a physiotherapy treatment (17\%) compared with those who did not (14\%). This suggests that most patients may cough and clear mucus independently without the need for specific interventions. Further studies are required to better elucidate the potential role of cough interventions in the postoperative period.

\section{Conclusions}

Our findings support the hypothesis that cough efficacy is impaired in the postoperative period after upper abdominal surgery. Postoperative restrictive lung dysfunction seems to be associated with this impairment. There was no significant association between peak cough flow and PPCs; however, cough impairment might result in clinically important consequences in a high-risk population. Future studies should investigate whether similar results are observed in specific patient subgroups (eg, those at higher risk for PPCs).

\section{ACKNOWLEDGMENTS}

We thank the fellow physiotherapists (Livia Kubagawa, Liane Shigemichi, Talita Stuchi, and Patrícia Teixeira) from the Respiratory Department of the Federal University of São Paulo for their assistance in data collection.

\section{REFERENCES}

1. Smetana GW, Lawrence VA, Cornell JE. Preoperative pulmonary risk stratification for noncardiothoracic surgery: systematic review for the American College of Physicians. Ann Intern Med 2006; 144(8):581-595.

2. Smith MC, Ellis ER. Is retained mucus a risk factor for the development of postoperative atelectasis and pneumonia? Implications for the physiotherapist. Physiother Theory Pract 2000;16(2):69-80.

3. Celli BR, Rodriguez KS, Snider GL. A controlled trial of intermittent positive pressure breathing, incentive spirometry, and deep breathing exercises in preventing pulmonary complications after abdominal surgery. Am Rev Respir Dis 1984;130(1):12-15.

4. King M, Brock G, Lundell C. Clearance of mucus by simulated cough. J Appl Physiol 1985;58(6):1776-1782.

5. Sancho J, Servera E, Díaz J, Marín J. Comparison of peak cough flows measured by pneumotachograph and a portable peak flow meter. Am J Phys Med Rehabil 2004;83(8):608-612.

6. Fiore JF Jr, Chiavegato LD, Denehy L, Paisani DM, Faresin SM. Do directed cough maneuvers improve cough effectiveness in the early period after open heart surgery? Effect of thoracic support and maximal inspiration on cough peak expiratory flow, cough expiratory volume, and thoracic pain. Respir Care 2008;53(8):1027-1034.

7. Jensen MP, Karoly P, Braver S. The measurement of clinical pain intensity: a comparison of six methods. Pain 1986;27(1):117-126.

8. Miller MR, Hankinson J, Brusasco V, Burgos F, Casaburi R, Coates A, et al. Standardisation of spirometry. Eur Respir J 2005;26(2):319338.

9. Pellegrino R, Viegi G, Brusasco V, Crapo RO, Burgos F, Casaburi $\mathrm{R}$, et al. Interpretative strategies for lung function tests. Eur Respir J 2005;26(5):948-968. 


\section{Cough Impairment After Open UpPer Abdominal Surgery}

10. Pereira ED, Fernandes AL, da Silva Anção M, de Araúja Pereres $C$, Atallah AN, Faresin SM. Prospective assessment of the risk of postoperative pulmonary complications in patients submitted to upper abdominal surgery. Sao Paulo Med J 1999;117(4):151-160.

11. Filardo Fde A, Faresin SM, Fernandes AL. [Index for a pulmonary postoperative complication after upper abdominal surgery: a validation study]. Rev Assoc Med Bras 2002;48(3):209-216. Article in Portuguese.

12. Machin D, Campbell MJ, Tan SB, Tan SH. Sample size tables for clinical studies, 3rd edition. Oxford: Wiley-Blackwell; 2008.

13. Swinscow TD. Correlation and regression. In: Statistics at Square One 1997. http://www.bmj.com/about-bmj/resources-readers/publica tions/statistics-square-one/11-correlation-and-regression. Accessed January 5, 2015.

14. Smith PR, Baig MA, Brito V, Bader F, Bergman MI, Alfonso A. Postoperative pulmonary complications after laparotomy. Respiration 2010;80(4):269-274.

15. Canet J, Gallart L, Gomar C, Paluzie G, Vallès J, Castillo J, et al. Prediction of postoperative pulmonary complications in a population-based surgical cohort. Anesthesiology 2010;113(6):1338-1350.

16. Paisani DM, Fiore JF Jr , Lunardi AC, Colluci DB, Santoro IL, Carvalho CR, et al. Preoperative six-min walking distance does not predict pulmonary complications in upper abdominal surgery. Respirology 2012;17(6):1013-1017.
17. Dilworth JP, Pounsford JC, White RJ. Cough threshold after upper abdominal surgery. Thorax 1990;45(3):207-209.

18. Sugimachi K, Ueo H, Natsuda Y, Kai H, Inokuchi K, Zaitsu A. Cough dynamics in oesophageal cancer: prevention of postoperative pulmonary complications. Br J Surg 1982;69(12):734-736.

19. Dureuil B, Cantineau JP, Desmonts JM. Effects of upper or lower abdominal surgery on diaphragmatic function. Br J Anaesth 1987; 59(10):1230-1235.

20. Joris J, Kaba A, Lamy M. Postoperative spirometry after laparoscopy for lower abdominal or upper abdominal surgical procedures. Br J Anaesth 1997;79(4):422-426.

21. Tokuda M, Tabira K, Masuda T, Nishiwada T, Shomoto K. Effect of modulated-frequency and modulated-intensity transcutaneous electrical nerve stimulation after abdominal surgery: a randomized controlled trial. Clin J Pain 2014;30(7):565-570.

22. American Association for Respiratory Care. AARC clinical practice guideline. Directed cough. Respir Care 1993;38(5):495-499.

23. Pasquina $P$, Tramèr MR, Granier JM, Walder B. Respiratory physiotherapy to prevent pulmonary complications after abdominal surgery: A systematic review. Chest 2006;130(6):1887-1899.

24. Mackay MR, Ellis E, Johnston C. Randomised clinical trial of physiotherapy after open abdominal surgery in high risk patients. Aust J Physiother 2005;51(3):151-159. 